02.2;09.1

\title{
Процессы возбуждения при столкновениях электронов с молекулами глутамина
}

\author{
(С) Н.М. Эрдевди, А.И. Булгакова , О.Б. Шпеник, А.Н. Завилопуло \\ Институт электронной физики НАН Украины, Ужгород, Украина \\ ฯ E-mail: alla.bulgakova.uzh@gmail.com
}

Поступило в Редакцию 13 марта 2020 г.

В окончательной редакции 18 мая 2020г.

Принято к публикации 18 мая 2020г.

\begin{abstract}
Проведены исследования процессов возбуждения при столкновениях электронов низких энергий $(1-100 \mathrm{eV})$ с молекулами глутамина в газовой фазе. Измерены оптические спектры излучения в диапазоне длин волн 250-520 nm. Установлено, что в результате распада молекул глутамина наиболее эффективно образуются молекулярные эмиссии ОН и некоторые другие молекулярные фрагменты, а также регистрируются возбужденные атомы водорода. Найдено, что энергии порогов возбуждения молекулярных эмиссий составляют $10-12 \mathrm{eV}$, в то время как для атомарных линий водорода $-13-15 \mathrm{eV}$. Представлены энергетические зависимости возбуждения отдельных эмиссий от порога возбуждения до $50 \mathrm{eV}$.
\end{abstract}

Ключевые слова: электрон, глутамин, возбуждение, фрагментация.

DOI: 10.21883/PJTF.2020.16.49852.18287

При столкновениях электронов с многоатомными молекулами (в том числе с биомолекулами) помимо упругого рассеяния в зависимости от энергии бомбардирующих электронов с различной вероятностью происходят процессы диссоциации, возбуждения самой молекулы, a также еe фрагментов, ионизации молекулы и ее фрагментов, причем в результате ионизации образуются как положительные, так и отрицательные ионы. Исследования этих процессов сегодня являются актуальной задачей. Среди методов исследований наиболее развиты методы масс-спектрометрии как положительных, так и отрицательных ионов [1]. Однако эти методы не дают информации о нейтральных фрагментах распада сложных молекул. Получение информации об этих фрагментах сопряжено с большими экспериментальными трудностями, связанными с регистрацией нейтральных частиц. Значительную информацию о диссоциации молекул можно получить, исследуя возбуждение образующихся фрагментов в результате распада молекулы под действием электронного удара. Нами была создана экспериментальная установка для изучения молекул в возбужденных состояниях и проведены эксперименты на молекулах некоторых основ нуклеиновых кислот.

Заметим, что взаимодействие низкоэнергетических (медленных) электронов с молекулами, которые входят в состав ДНК, РНК и их фрагментов, является предметом интенсивных исследований [1-4]. В первую очередь внимание уделяется установлению механизма процессов, приводящих к повреждениям структуры этих молекул и вызывающих гибель отдельных клеток и организма в целом. Часто проблема сводится к базовым составляющим ДНК и РНК, поскольку точное понимание процессов, происходящих на молекулярном уровне, рассматривается как важный шаг к описанию более сложных явлений, происходящих на клеточном уровне.

Процесс возбуждения молекул является одним из наиболее важных элементарных процессов, происходящих в молекулах при их взаимодействии с медленными электронами. Этот процесс сопровождается переходом одного или нескольких электронов исходной молекулы на вышестоящие электронные состояния с последующим излучением, которое может охватывать широкий диапазон длин волн от инфракрасного до видимого и ультрафиолетового [5]. Процессы возбуждения сопровождаются излучением спектральных полос молекул и их фрагментов, поэтому наиболее эффективным методом их исследования является оптический.

Использование моноэнергетических пучков электронов для возбуждения сложных молекул в газовой фазе позволяет получить информацию о положении и структуре энергетических уровней, а также оценить относительную вероятность процесса возбуждения. Исследования флуоресценции и энергетических зависимостей возбуждения электронно-колебательных состояний позволяют судить об эффективности преобразования внутренней энергии в сложных молекулах в оптическое излучение. Несмотря на безоговорочную значимость изучения основных механизмов структурных изменений в молекулах аминокислот под действием низкоэнергетических электронов, данных такого рода мало [6].

Цель настоящей работы состоит в исследовании спектров излучения молекул глутамина в газовой фазе при возбуждении электронами низких энергий, измерении оптических функций возбуждения (ОФВ) отдельных эмиссий излучения и установлении механизмов их возбуждения. 
Эксперименты проводились спектроскопическим методом с регистрацией спектров оптического излучения паров исследуемого вещества, возбужденного электронами фиксированной энергии. Нами был использован метод паронаполненной ячейки [7], в которой создавалась необходимая концентрация молекул глутамина в области взаимодействия с электронами. Контейнер с исследуемыми молекулами располагался в непосредственной близости к ячейке и имел независимый подогрев. Температура контейнера поддерживалась равной $70 \pm 3^{\circ} \mathrm{C}$. Температура элементов конструкции электронной пушки и ячейки столкновений поддерживалась на $20-30^{\circ} \mathrm{C}$ выше температуры контейнера. Давление остаточных газов в вакуумной камере в рабочих условиях не превышало $1.3 \cdot 10^{-4} \mathrm{~Pa}$.

Пучок электронов диаметром $2 \mathrm{~mm}$, формируемый четырехэлектродной пушкой с оксидным катодом, с силой тока $\sim 20 \mathrm{~mA}$ в интервале энергий $1-100 \mathrm{eV}$ проходит паронаполненную ячейку и детектируется цилиндром Фарадея. Нестабильность тока электронов, прошедших камеру столкновений, не превышала 3\%. Моноэнергетичность электронного пучка (полная ширина на полувысоте) была не хуже $\sim 0.5 \mathrm{eV}$ и определялась с помощью специальной программы управления экспериментом перед каждым измерением. Энергия электронов при измерениях ОФВ задавалась и сканировалась автоматически с шагом 100-400 $\mathrm{meV}$.

Излучение из области взаимодействия электронов с молекулами направлялось на входную щель дифракционного монохроматора МДР-2 и детектировалось фотоэлектронным умножителем ФЭУ-106. Необходимая точность измерения полезного сигнала в зависимости от его величины обеспечивалась выбором экспозиции накопления сигнала, которая в каждой точке составляла 10-40 s. Оптические спектры излучения в диапазоне длин волн $\lambda=250-520 \mathrm{~nm}$ записывались с шагом $0.814 \mathrm{~nm}$, при этом ширина щелей монохроматора $1 \mathrm{~mm}$ обеспечивала разделение $\Delta \lambda=2 \mathrm{~nm}$. Для исключения влияния фона засветки от катода на величину полезного сигнала электронный пучок модулировался и фон вычитался из общего сигнала.

Важным фактором корректной интерпретации результатов измерений является калибровка энергетической шкалы пучка возбуждающих электронов. В наших опытах она осуществлялась двумя методами: 1) по смещению вольт-амперной характеристики электронного тока пучка на коллектор; 2) по положению известного резко выраженного максимума на ОФВ спектральной линии молекулы азота $337.1 \mathrm{~nm}\left(C^{3} \Pi_{u} \rightarrow B^{3} \Pi_{g}\right)$. Разность в энергетическом положении этого максимума $(14.2 \mathrm{eV}[8])$ и измеренной нами кривой составляла контактную разность потенциалов. Таким образом, установленная после калибровки шкала энергий электронов при измерениях ОФВ была не хуже $\pm 0.1 \mathrm{eV}$.

Остановимся на результатах наших исследований процессов элекронного возбуждения молекул глутамина (2-аминопентанамид-5-овая кислота), который является

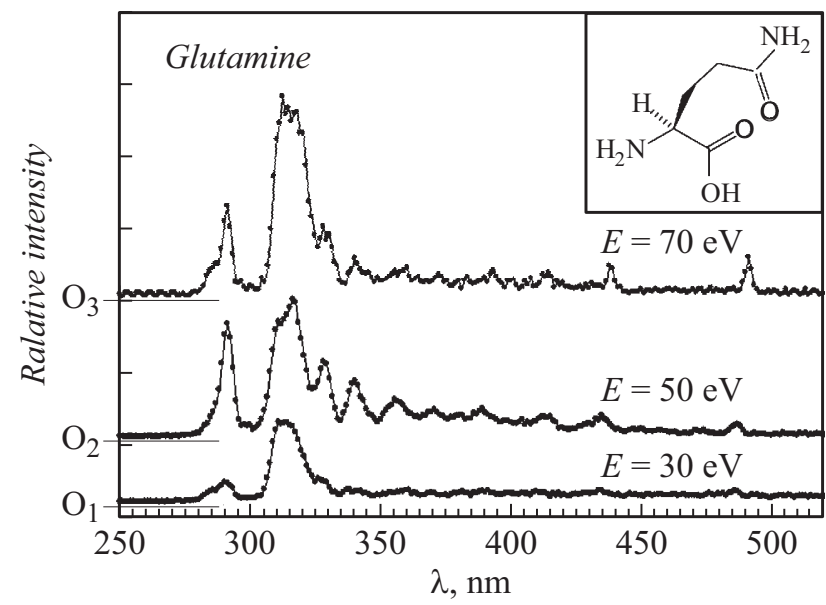

Рис. 1. Спектры излучения молекулы глутамина. На вставке - структурная схема молекулы глутамина.

одним из ярких представителей 20 стандартных аминокислот, входящих в состав белков. Как следует из структурной схемы глутамина (вставка на рис. 1), он содержат аминную $\left(-\mathrm{NH}_{2}\right)$ и карбоксильную $(-\mathrm{COOH})$ группы. При этом карбоксильная группа может вращаться, а атом водорода может ориентироваться как в направлении азота, так и в обратном направлении. Кроме того, конформационная изменчивость молекул способствует переориентировке гибкой карбоксильной $(-\mathrm{COOH})$ и аминной $\left(-\mathrm{NH}_{2}\right)$ групп, при этом образуются различные внутримолекулярные водородные связи, которые, например, соединяют неразделенную пару атомов азота с водородом гидроксильной группы (N...HO) или же устанавливают связь между атомом водорода аминной группы и атомом кислорода карбонильной $(\mathrm{NH} . . \mathrm{O}=\mathrm{C})$ либо гидроксильной (NH. . . OH) групп.

На рис. 1 приведены спектры излучения молекулы глутамина при энергиях бомбардирующих электронов 30,50 и $70 \mathrm{eV}$. Для определения абсолютной интенсивности отдельных полос и линий необходима постановка специального эксперимента. Однако если кривые нормировать на пик, соответствующий максимуму излучения линии $306.4 \mathrm{~nm}$, то, как видно из рис. 1, полученные спектры существенно зависят от энергии бомбардирующих электронов. Так, если при энергии электронов $30 \mathrm{eV}$ выделяются только одна довольно интенсивная полоса эмиссии при 300-320 nm и незначительная при 280-300 nm, то при энергиях электронов 50 и $70 \mathrm{eV}$ кроме полосы эмиссии 300-320 nm наблюдается набор из ряда отдельных узких полос и линий в длинноволновой области спектра. С использованием результатов работ $[9,10]$ удалось идентифицировать бо́льшую часть наблюдаемых полос и линий эмиссий. Так, линии эмиссии 486, 436, $410 \mathrm{~nm}$ можно отождествить с линиями атома водорода серии Бальмера. Широкая полоса 300-320 nm однозначно соответствует распаду резонансного состояния радикала $\mathrm{OH}^{*}\left(2 \Pi_{i}(X) \leftarrow{ }^{2} \Sigma^{+}(A)\right)$, энергия воз- 

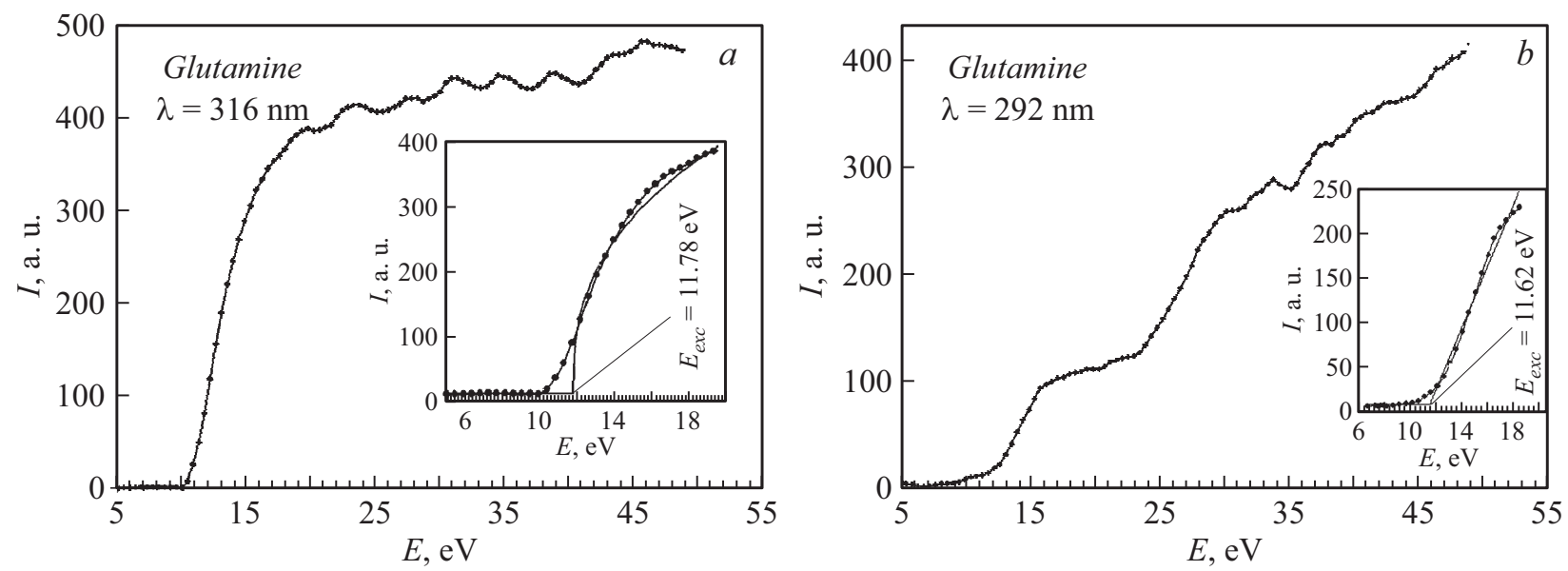

Рис. 2. Оптические функции возбуждения молекулы глутамина: $a-\lambda=316 \mathrm{~nm}, b-\lambda=292 \mathrm{~nm}$. На вставках приведены пороговые участки ОФВ: точки - эксперимент, сплошная линия - аппроксимация.

Пороги возбуждения и положение особенностей на функциях возбуждения эмиссий молекулы глутамина

\begin{tabular}{c|c|c}
\hline $\begin{array}{c}\text { Длина волны, } \\
\mathrm{nm}\end{array}$ & $\begin{array}{c}\text { Порог возбуждения, } \\
\mathrm{eV}\end{array}$ & $\begin{array}{c}\text { Положение особенностей, } \\
\mathrm{eV}\end{array}$ \\
\hline 292 & $11.62 \pm 0.2$ & $29.5,33.5,32.5$ \\
310 & $11.66 \pm 0.25$ & $17.5,20,22.5,28.5,32,36,41$ \\
316 & $11.78 \pm 0.25$ & $19.5,23,27,31,34,38.5,43.5,45$ \\
329 & $9.09 \pm 0.2$ & $10.9,25,30.5,37,40.5$ \\
340 & $12.49 \pm 0.25$ & 17 \\
436 & $4.39 \pm 0.2$ & $10,16.5,39,44.5$ \\
& $4.70 \pm 0.2$ & $7.5,14,18.5,25.3,43,46.5$
\end{tabular}

буждения которого равна $4.06 \mathrm{eV}$, при этом полоса с длиной волны $306.4 \mathrm{~nm}$ принадлежит фрагменту ОН*. Линия эмиссии $278 \mathrm{~nm}$ может быть связана с возбуждением и распадом ${ }^{2} \Sigma^{+}(B)$-состояния радикала $\mathrm{OH}^{*}$ $\left(E_{\text {exc }}=8.66 \mathrm{eV}\right)$. Остальные линии и полосы эмиссии в области 280-330 nm, скорее всего, также принадлежат фрагментам $\mathrm{OH}^{*}$.

Таким образом, можно заключить, что при столкновении электронов с молекулами глутамина возможны различные каналы распада, наиболее вероятными из которых являются отрыв одного из атомов водорода или фрагмента ОН в возбужденном состоянии с последующим излучением.

Для дальнейшего выяснения механизмов распада исходной молекулы глутамина при столкновении с электронами нами изучены энергетические зависимости (рис. 2) возбуждения отдельных эмиссий (полос, линий) и определены их пороги возбуждения (см. таблицу) с помощью методики, описанной в [4]. Начальные энергии возбуждения эмиссий определялись по пороговым участкам ОФВ, которые представлены на вставках к рис. 2.

Примечательной особенностью представленных на рис. 2 ОФВ эмиссий гидроксильной группы ОН $(\lambda=316$ и $292 \mathrm{~nm})$ является различная динамика роста с увеличением энергии возбуждающих электронов, а также наличие целого ряда особенностей и изломов, положение которых приведено в таблице. Как видно из рисунка, пороги возбуждения этих эмиссий близки между собой $(\sim 11 \mathrm{eV})$, но характер их возбуждения существенно различен. Функция возбуждения $\lambda=316 \mathrm{~nm}$ имеет резко выраженный рост интенсивности у порога с последующим выходом на плато при энергии $18-20 \mathrm{eV}$. Что касается ОФВ $\lambda=292 \mathrm{~nm}$, то она имеет замедленный рост у порога, небольшой пологий максимум при $18 \mathrm{eV}$ и далее почти линейное возрастание интенсивности с увеличением энергии бомбардирующих электронов. Иначе ведут себя функции возбуждения линий водорода $(\lambda=486$ и $436 \mathrm{~nm})$ : пороги возбуждения этих линий практически совпадают с энергией возбуждения исходных уровней соответствующих линий атома водорода $(\sim 12-13 \mathrm{eV})$, затем с увеличением энергии возбуждающих электронов наблюдается плавный рост интенсивности излучения линий. На основе изложенного выше, с одной стороны, можно предположить, что при столкновениях электронов с молекулами глутамина происходит отрыв одного из атомов водорода по схеме

$$
\mathrm{C}_{5} \mathrm{H}_{10} \mathrm{~N}_{2} \mathrm{O}_{3}+e^{-} \rightarrow \mathrm{C}_{5} \mathrm{H}_{9} \mathrm{~N}_{2} \mathrm{O}_{3}+\mathrm{H}^{*}+e^{-},
$$

a с другой - предположить, что при этом в процессе столкновения образуется сверхвозбужденная молекула 
глутамина [11] с последующим распадом на возбужденные состояния фрагментов по схеме

$$
\begin{aligned}
& \mathrm{C}_{5} \mathrm{H}_{10} \mathrm{~N}_{2} \mathrm{O}_{3}+e^{-} \rightarrow\left(\mathrm{C}_{5} \mathrm{H}_{10} \mathrm{~N}_{2} \mathrm{O}_{3}\right)^{* *} \\
& +e^{-} \rightarrow \mathrm{C}_{5} \mathrm{H}_{9} \mathrm{~N}_{2} \mathrm{O}_{3}+\mathrm{OH}^{*}+e^{-}
\end{aligned}
$$

В заключение отметим, что прямой отрыв атома водорода и другие механизмы фрагментации могут свидетельствовать о том, что исходная молекула глутамина распадается в момент взаимодействия с электроном. Более детальный анализ обнаруженных нами особенностей на ОФВ требует специального теоретического исследования.

\section{Благодарности}

Авторы выражают благодарность Е.Э. Контрошу за помощь при выполнении настоящей работы.

\section{Конфликт интересов}

Авторы заявляют, что у них нет конфликта интересов.

\section{Список литературы}

[1] Sanche L. // Eur. Phys. J. D. 2005. V. 35. P. 367-390.

[2] Якубке Х.Д., Ешкайт Х. Аминокислоты. Пептиды. Белки. М.: Мир, 1985. 75 c.

[3] Завилопуло А.Н., Булгакова А.И. // Письма в ЖТФ. 2019. T. 45. B. 24. C. $36-40$.

[4] Zavilopulo A.N., Shpenik O.B., Mylymko A.N., Shpenik V.Yu. // Int. J. Mass Spectr. 2019. V. 441. P. 1-7. https://doi.org/10.1016/j.ijms.2019.03.008

[5] Chatterley A.S., Johns A.S., Stavros V.G., Verlet J.R.R. // J. Phys. Chem. A. 2013. V. 117. P. 5299-5305.

[6] McConkey J.W., Malone C.P., Johnson P.V., Winstead C., McKoy V., Kanik I. // Phys. Rep. 2008. V. 466. P. 1-103.

[7] Эрдевди Н.М., Звенигородский В.В., Шпеник О.Б., Романова Л.Г. // Оптика и спектроскопия. 2013. Т. 114. № 1. C. $51-56$.

[8] Скубенич В.В., Запесочный М.П. // Химия высоких энергий. 1975. Т. 9. № 5. С. 387-394.

[9] Soskida M.T.I. // Uzhhorod University Scientific Herald. Ser. Physics. 2004. N 16. P. 54-58.

[10] Вуль А.Я., Кидалов С.В., Миленин В.М., Тимофеев Н.А., Ходорковский М.А. // Письма в ЖТФ. 1999. Т. 25. В. 1. C. $10-16$.

[11] Wasowicz T.J., Kivimäki A., Coreno M., Zubek M. // J. Phys. B. 2012. V. 45. P. 205103. https://doi.org/10.1088/0953$4075 / 45 / 20 / 205103$ 